\title{
Scalar Field Dark Matter: head-on interaction between two structures
}

\author{
Argelia Bernal ${ }^{1}$ and F. Siddhartha Guzmán ${ }^{2}$ \\ ${ }^{1}$ Departamento de Física, Centro de Investigación y de Estudios Avanzados del IPN, AP 14-740,07000 México D.F., México. \\ ${ }^{2}$ Instituto de Física y Matemáticas, Universidad Michoacana de San Nicolás de Hidalgo. Edificio C-3, \\ Cd. Universitaria, C. P. 58040 Morelia, Michoacán, México.
}

(Dated: June 12, 2018)

\begin{abstract}
In this manuscript we track the evolution of a system consisting of two self-gravitating virialized objects made of a scalar field in the newtonian limit. The Schrödinger-Poisson system contains a potential with self-interaction of the Gross-Pitaevskii type for Bose Condensates. Our results indicate that solitonic behavior is allowed in the scalar field dark matter model when the total energy of the system is positive, that is, the two blobs pass through each other as should happen for solitons; on the other hand, there is a true collision of the two blobs when the total energy is negative.

PACS numbers: 04.40.-b 05.45.Yv 05.30.Jp 04.25.Dm, 98.62.Gq
\end{abstract}

\section{INTRODUCTION}

The most widely studied dark matter hypothesis consists in assuming that it is made of point-like cold particles that are responsible for the formation of structure in the universe; among the most studied candidates nowadays are the supersymmetric particles that would behave as a cold fluid made of particles. However, two problems associated to the point-like nature of dark matter are that the resulting gravitational collapse shows a central density profile that is not flat and on the other hand it predicts a non-observed amount of small structures. An alternative to ameliorate these two problems consists in assuming that the dark matter is made of an ultralight spinless particle, the so called Scalar Field Dark Matter Model (SFDM). In the cosmological frame, the analysis of such hypothesis indicated that the power of structures could be controlled through a parameter in the model, that is, the mass of the scalar field representing the spinless particle [1, 2, 3, 4]. Once the mass $m$ of the boson is fixed the power spectrum suffers a cutt-off according to the mass of the smallest structure desired. An interesting assumption in such analysis is that the scalar field potential was a cosh-like potential, that behaved as an exponential at early times and as a free field case (quadratic potential) at late times, whose behavior was that of the usual cold dark matter model. Moreover, it was found that the SFDM enjoys the same advantages at cosmic scale as the standard lambda cold dark matter model.

Because the SFDM requires the existence of a fundamental scalar field for its reliability, it is natural to consider that this scenario fits very well within unification theory scenarios and braneworld models [5]. This by itself is a good enough reason to consider the SFDM as an alternative powerful model, because it contains intrinsically the spinless boson as dark matter particle. However, once at cosmic scales the model matches with observations, it is necessary to study the predictions of the model at structure scales. In this sense there have been several results indicating that the model is good also at galactic scales and here we briefly summarize such results.

At the early stages of the galactic dark matter model, fully general relativistic stationary solutions were proposed to explain the phenomena like the flatness of rotation curves, assuming the scalar field was real [6, 7], non-topological scalar field dark halos [8] complex scalar fields [9] and global monopoles [10]. Later on, the assumptions relaxed to the newtonian limit of such general relativistic models. By the time, quintessential dark matter halos [11, 12, 13] and the fluid dark matter made of scalar fields was proposed as an alternative galactic dark matter model [14] and the collapse of fuzzy dark matter made of a scalar field was analyzed in one dimension [15]. On the other hand, the assumption of time independence was also relaxed and scalar field dark matter halos were proposed to be gigantic Oscillatons, that is, time dependent fully relativistic scalar field solutions to the Einstein-Klein-Gordon system of equations [16, 17].

Currently what appears to be the interesting case is that of the time dependent newtonian limit of the model, that is, the Schrödinger-Poisson (SP) system of equations would describe the model at local scales. In this direction relevant results have been found, for instance: it was shown that when the evolution of a structure of galactic mass is followed after the turnaround point, it quickly virializes and tends to a stationary equilibrium solution of the SP system of equations, whereas one of the size of a supercluster would still be relaxing at the present time 18]; the condition is that the mass of the boson $\left(m \sim 10^{-23} \mathrm{eV}\right)$ is the one that better cuts-off the power spectrum at galactic scales as shown in [1, 2, 3]. Thus, at the moment the pieces of the model seem to match both, at cosmic and at local scales. In fact, recently in [19] it was shown that the scalar field gravitational collapse tolerates the introduction of a self-interaction term in the potential, which makes the model to seem quite like a self-gravitating Bose-Condensate. In [20] we showed that spherically symmetric equilibrium solutions of the SP system are stable against non-spherical perturbations, and moreover, such configurations played the role of late-time attractors for initially quite general ax- 
isymmetric initial density profiles.

What we present here is a step forward in the direction of studying the evolution of scalar field structures. We perform numerical studies of scalar binary configurations, as a first step towards the making of a numerical code with no symmetries and for N-scalar objects. These studies would tell us about possible restrictions on selfinteraction terms for the scalar field, and the way single configurations interact with each other. We restrict ourselves to the case of head-on interaction, which can be handled with a 2D code with axi-symmetry. We choose to write down the SP in cylindrical coordinates:

$$
\begin{aligned}
i \frac{\partial \psi}{\partial t} & \left.=-\frac{1}{2}\left(\frac{\partial^{2} \psi}{\partial x^{2}}+\frac{1}{x} \frac{\partial \psi}{\partial x}+\frac{\partial^{2} \psi}{\partial z^{2}}\right)+U \psi+\Lambda|\psi|^{2} \psi 1\right) \\
\frac{\partial^{2} U}{\partial x^{2}} & +\frac{1}{x} \frac{\partial U}{\partial x}+\frac{\partial^{2} U}{\partial z^{2}}=\psi^{*} \psi
\end{aligned}
$$

where $\psi=\psi(x, z, t)$ and $U=U(x, z, t)$ are the wave function and the gravitational potential respectively; $x, z$ are the radial and axial cylindrical coordinates respectively. The third order term in Eq. (1) is related to a self-interacting term, in which $\Lambda$ corresponds to the swave scattering length in the Gross-Pitaevskii approximation for Bose Condensates [21]. This term was shown to play the role of determining the compactness of the structure [19]. Equations (112) use the units and scaling $\hbar=c=1$ with $x \rightarrow m x, z \rightarrow m z, t \rightarrow m t$ and the wave function $\psi \rightarrow \sqrt{4 \pi G} \psi$, where $m$ is the mass of the ultralight boson. As a consequence of this change of units is that the mass of a system will be in units of $[M]=M_{p l}^{2} / m$ as found also for fully relativistic boson star solutions [19, 22]; this implies that the value $m$ sets the physical lenght and time scale of the configuraions evolved. This mass, together with the scaling relations of the Schrödinger-Poisson system [18, 20, 23] are the basics for transforming back to physical units the system of interest (see an exmple below).

The paper is organized as follows. In the next section we briefly describe the code used. In section [II] we construct initial data containing two spherically symmetric equilibrium configurations along the $z$ axis. In section IV] we show the results for the head-on interaction of the two structures. Finally in section $\square$ we draw some conclusions.

\section{NUMERICAL METHODS}

The evolution. The most common numerical technique for time-integrating Eq. (11) is implicit with alternating direction splitting of the evolution operator [24, 25]. The reason for this is that the evolution operator is unitary. Nevertheless, we used such method in [23], where no need for splitting the operator on the right hand side of Eq. (11) was needed; in [19, 20] it was shown that explicit methods preserve also the number of particles and no significant difference in the results is found after using one method or the other. For the present case an explicit approximation of the full implicit method (in practice, a modified iterative Crank-Nicholson method [17]), with second order finite differencing to calculate the spatial derivatives is used. The reason to avoid using the implicit method is the difficulty to reduce the evolution operation to a tridiagonal system of equations when considering a non-zero $\Lambda$, which makes the Schrödinger equation a non-linear one, a situation not descussed in [24, 25].

Poisson equation. Equation (2) is an elliptic equation for $U$ which we solve using the 2D five-point stencil for the derivatives and a successive over-relaxation (SOR) iterative algorithm with optimal acceleration parameter (see e.g. [26] for details about SOR). In order to impose boundary conditions we made sure the boundaries were far enough for the mass $M=\int|\psi|^{2} d^{3} x$ to be the same along the three faces of the domain and used the monopolar term of the gravitational field; that is, we used the value $U=-M / r$ along the boundaries with $r=\sqrt{x^{2}+z^{2}}$. At the axis we demanded the gravitational potential to be symmetric with respect to the axis.

We use a sponge in the outermost region of the domain. The sponge is a concept used with success in the past when dealing with the Schrödinger equation (for detailed analyses see [23, 27]). This technique consists in adding up to the potential in the Schrödinger equation an imaginary part. The result is that in the region where this takes place there is a sink of particles, and therefore the density of probability approaching this region will be damped out, with which we get the effects of a physically open boundary.

Basic testbeds of this code evolving single equilibrium configurations can be found in [20], where the results are also compared with previous studies with spherical symmetry and linear perturbation theory.

\section{INITIAL DATA}

Details about the construction of initial data for spherically symmetric equilibrium configurations can be found in [19, 20, 23]. Here we briefly mention the procedure used in our binary case. What we do is to superpose two spherically symmetric ground state equilibrium configurations upon the same $2 \mathrm{D}$ axially symmetric grid, whose construction is described as follows. In spherical symmetry equations (12) read

$$
\begin{aligned}
i \partial_{t} \psi & =-\frac{1}{2 r} \partial_{r}^{2}(r \psi)+U \psi+\Lambda|\psi|^{2} \psi \\
\partial_{r}^{2}(r U) & =r \psi \psi^{*} .
\end{aligned}
$$

where $r=\sqrt{x^{2}+z^{2}}$. If a time dependence of the type $\psi=\phi e^{i \omega t}$, regularity at the origin, and an isolation con- 
dition $\phi(r \rightarrow \infty)=0$ are assumed, the system becomes an eigenvalue problem for $\phi$ with eigenvalue $\omega$ :

$$
\begin{aligned}
\partial_{r}^{2}(r \phi) & =2 r(U-\omega)+2 \Lambda|\phi|^{2} \phi, \\
\partial_{r}^{2}(r U) & =r \phi^{2} .
\end{aligned}
$$

In order to solve these equations we discretize them and use a shooting method that bisects the value of $\omega$ so that the boundary conditions hold with certain desired accuracy. The solutions constructed in this way are called equilibrium configurations. For each value of $\Lambda$ it is possible to construct the whole branch of equilibrium configurations as shown in [19]. However there is an extra ingredient in these solutions: the number of nodes of the wave function. When the wave function is nodeless we say it belongs to the ground state; when the wave function has a given number of nodes we can construct also the branches of solutions that -by analogy with the particle in a box- are called excited spherical states. However, as shown in [19, 23] such excited states are unstable and decay into ground states; in fact ground states are stable and late-time attractors for quite arbitrary initial wave function profiles [19, 20]. Because the excited states are unstable and decay in a short time scale we collide in the present task only ground state configurations.

Once we account with these ground state data: i) we interpolated the wave function of such configuration centered at $\left(0, z_{0}\right)$, ii) we place another of these equilibrium configuration at the point $\left(0,-z_{0}\right)$, iii) we choose $z_{0}$ so that the two configurations are far enough one from the other (see below) and iv) resolved Poisson equation (2). Then we have initial data for two ground state equilibrium configurations in our axially symmetric domain.

Summarizing, we choose to solve the initial value problem in spherical coordinates to make sure that we start the evolution with very accurate values, and we evolve in a 2D grid using cylindrical coordinates because we found them necessary and practical for the binary case we are interested in.

Special warning is needed in Eq. (11), because it is nonlinear for the $\Lambda \neq 0$ case, which indicates that the superposition of two wave functions is not allowed. Assume $\psi_{1}$ and $\psi_{2}$ represent the solutions of the initial spherically symmetric configurations that are to be superposed onto the $2 \mathrm{D}$ grid; the density of probability in (2) for the total wave function $\psi=\psi_{1}+\psi_{2}$ is $\left|\psi_{1}+\psi_{2}\right|^{2}$ and unless these states are orthogonal one cannot consider the naive superposition above is allowed. Thus we choose the distance between the configurations such that the interference (given by the scalar product of the two wave functions) $<\psi_{1}, \psi_{2}>$ is of the order of the precision of our calculations, say, in our case, the precision of the interpolation of the data into the $2 \mathrm{D}$ grid. Thus we can think of the system as one made of two adequately superposed equilibrium configurations we want to collide. An example of the interference term is shown in Fig. 1.

The superposition of configurations by itself would say little about whether or not two configurations collide.
We add an extra ingredient to the system, that is, an initial head-on momentum to the initial scalar field balls. We simply generate different initial kinematical states by assigning new values to the wave functions of the equilibrium configurations: $\psi_{1} \rightarrow \psi_{1} e^{i p_{z} z}$ and $\psi_{2} \rightarrow \psi_{2} e^{-i p_{z} z}$. The resulting physical situation involves a considerable change in the value of the expectation value of kinetic energy in the system.
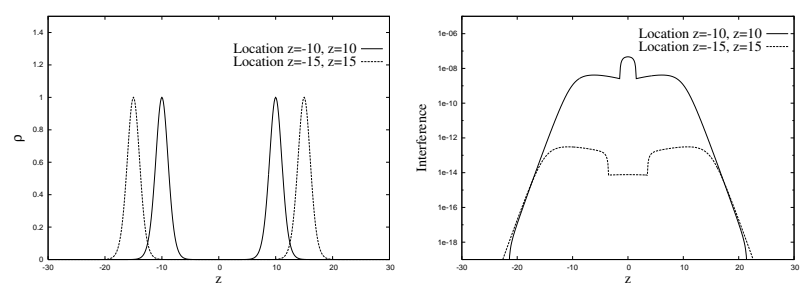

FIG. 1: (Left) An example of two ground state configurations superposed along the $z$-axis with two different separations. (Right) The interference $\left\langle\psi_{1}, \psi_{2}\right\rangle$ is shown for two cases. Different separations are used and the two configurations have a central field value $\psi(0)=1.0$. The initial head-on momentum is $p_{z}=3.0$.

Fortunately, in the present Newtonian low energy regime it is possible to estimate expectation values of physical quantities, a property difficult to pose in the fully general relativistic case. For instance, we account with the observables that allow one to monitor the evolution of the physical situation. Because we deal with a quantum mechanical system, we simply estimate the expectation values of the following interesting operators:

$$
\begin{aligned}
K & =-\frac{1}{2} \int \psi^{*} \nabla^{2} \psi d^{3} x \\
W & =\frac{1}{2} \int \psi^{*} U \psi d^{3} x \\
I & =\int \Lambda|\psi|^{4} d^{3} x
\end{aligned}
$$

which are the expectation values of the kinetic, gravitational and self-interaction energies. These quantities are quite important at determining the state of the system at any time during the evolution of the system. That is, the value of the total energy $E=K+W+I$ indicates whether we account with a bounded system or not, and the very important virial theorem relation $2 K+W+3 I=0$ [28], which is nearly satisfied when the system gets virialized and relaxed through whatever channels available, for instance, the emission of scalar field bursts, the so called gravitational cooling. 


\section{HEAD-ON COLLISIONS AND SOLITONIC BEHAVIOR}

\section{A. Equal mass case}

The first scenario one might think of is the collision of two equal mass ground state configurations. In Fig. 2 we show snapshots of the density profile along the $z$-axis for an initial configuration with $p_{z}=3.0$ and $z_{0}=15$ in the free field case $(\Lambda=0)$. What is found is that the two blobs move toward each other and eventually they lie upon each other, an interference pattern gets formed and after a while one blob moves toward the left and the other one toward the right. The first interpretation is that the initial data behave like solitons. Unfortunately we cannot be confident about the solitonic behavior because the shape of the blobs gets deformed after the "collision" and an increase of the amplitude and shrink in the width are manifest. After the distributions approach the boundary (located at $z= \pm 30$ ) the density of probability is absorbed by the sponge and its integral $M$ drops to zero. At this point we are unable to track the evolution further in time and we ignore whether the blobs might return and collide again and repeat such process as many times as desired until there is energy released (e.g. through the emission of scalar field) and the encounters get damped allowing eventually a true collision. In Fig. 3 we show a zoom of the interference pattern at the time when superposition of the configurations around $t \sim 5$ occurs.

Of course, not all the initial configurations constructed present this behavior and we have found that a criterion to decide whether this behavior is allowed or not is the value of the total energy $E=K+W+I$. In Figs. 4 and 5 we show the total energy of different types of initial configurations. In Fig. 4 we present different situations for the free field case $\Lambda=0$ and two particular cases: $p_{z}=1.0$ and $p_{z}=0$; in the first case the solitonic behavior is achieved and the total energy is always positive and approaches zero because the density of probability has left the numerical domain; in the second case the total energy is always negative and at the end of the day what is found is that there is a single blob in the middle, indicating that the system is oscillating around a bounded object. We show snapshots of this behavior later when dealing with the more interesting unequal mass case. About the other cases in this plot $p_{z}=0.75,0.71,0.7$ we cannot decide whether they show solitonic behavior or not in the time we used to run our simulations and we can only observe that the density profiles are severely distorted by the collision.

In Fig. 5 we show the same criterion for configurations with the self-interaction term $(\Lambda=0.2)$. Again, when the total energy is extremely positive or extremely negative we are able to decide whether the configurations collide or they trespass each other.

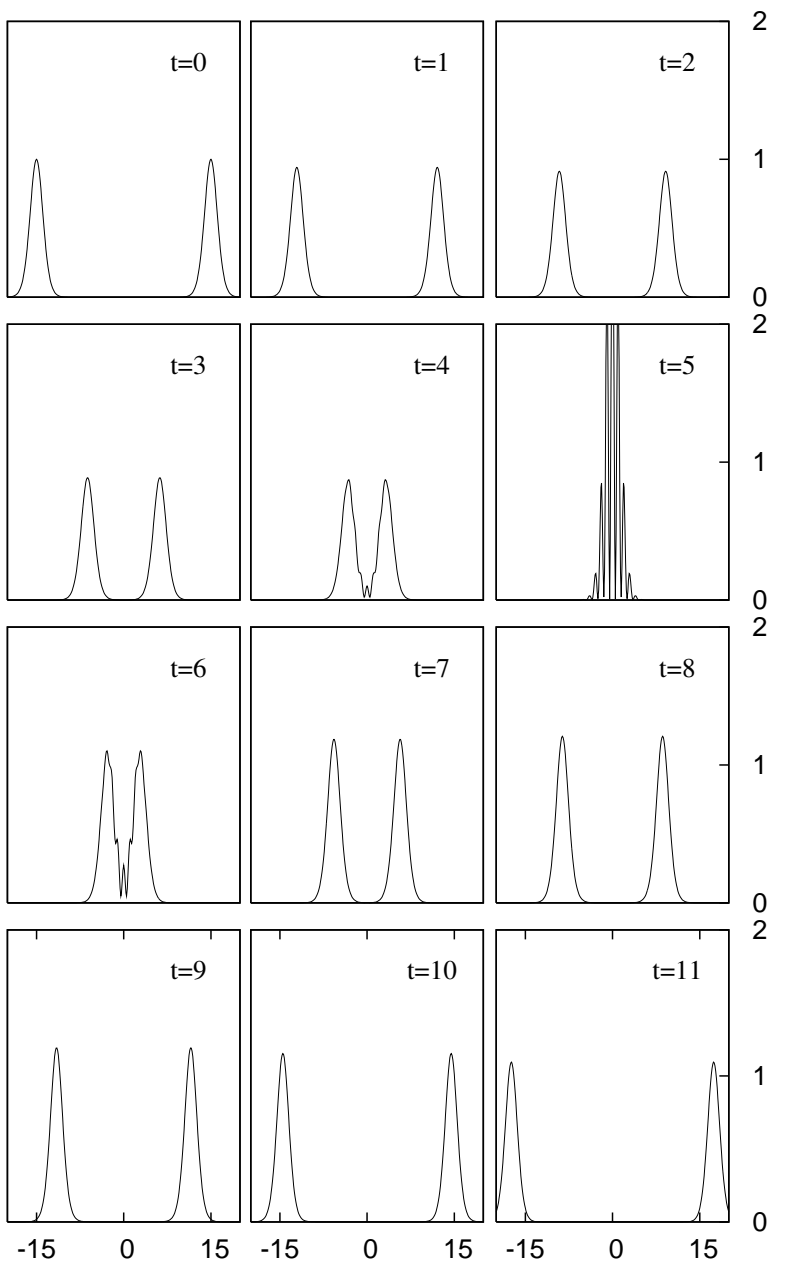

FIG. 2: Snapshots of the density of probability for interaction between two initial configurations with $\Lambda=0, p_{z}=3.0$ and $z_{0}=15$. The configuration shows solitonic behavior due to the fact that the total energy is always positive of the order of $E \sim 32$ until the blobs get absorbed by the sponge. The numerical domain used is $x \in[0,30], z \in[-30,30]$ with resolution $\Delta x=\Delta z=0.125$.

\section{B. Unequal mass case}

The unequal mass case helps at deciding whether the configurations in the above examples truly trespass each other or bounce. In fact up to now it is not possible to say anything about this because of a few reasons: i) the expectation absolute value of the linear momentum along the head-on direction is equal for both half planes $z>0$ and $z<0$, ii) the mass in each half plane is also the same in both semiplanes, iii) the expectation value of the linear momentum in the head-on direction is zero all the time.

In the unequal mass case we have the advantage of being able to distinguish the half planes masses and linear momentum. In Fig. 6] we show snapshots of the unequal mass case for $\Lambda=0.2$ and initial parameters $p_{z}=3.0$ 


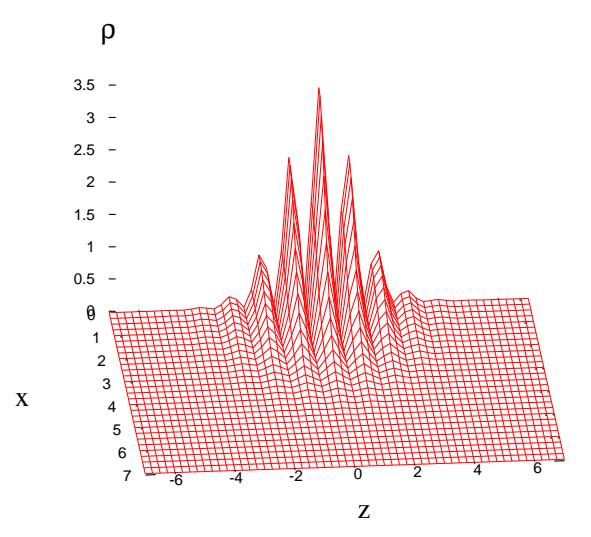

FIG. 3: The pattern of interference formed during the collision of two configuration with $\Lambda=0, z_{0}=15, p_{z}=3.0$ (the same case as in the previous figure). After this stage, the two blobs continue their way in the initial direction and behave as solitons (see Fig. 22). They are not strict solitons because they slightly deform during the process.

and $z_{0}=15$ that show solitonic behavior with $E>0$ all the way. It can be seen clearly that the initial blobs are actually trespassing each other although they suffer a profile deformation until the wave function reaches the sponge region.

In Fig. 7 we show the mass transfer from the $z>0$ to the $z<0$ half planes and viceversa; notice that the mass transfers from one side to the other in a very effective way. We also show the expectation value of the linear momentum along $z$ in both half planes; what is found is that the momentum is also transfered from one side to the other. The perfect solitonic behavior would consist of having these two properties plus the unachieved one related to the preservation of the density profile.

\section{An example of collision}

As a final result we show what happens when an initial configuration presents a negative total energy. In Fig. 8 we show the density profile along $z$ for a collision case corresponding to $\Lambda=0, z_{0}=10$ and $p_{z}=0$, that is, only the pure gravitational force drives the dynamics of the binary. It can be observed that the blobs actually merge and remain sitting on a fixed point around the center of mass, the density tends to get stabilized, the virial relation starts oscillating aorund zero with smaller amplitude, the total energy starts stabilizing, so as the mass of the system.

In order to ullistrate what our results mean in physical units we use the run in Fig. 8 to estimate the time-scale for the collision of binary equal mass head-on case. We start from the fact that the mass of ground state configurations is $M \sim 10^{11} M_{\odot}$ and the mass of the boson is $m=10^{-23} \mathrm{eV}$; the separation is $20 \sim 3.52 \mathrm{kpc}$ and the time the density peak is maximum is $t_{\text {collision }} \sim 52.5 \sim$

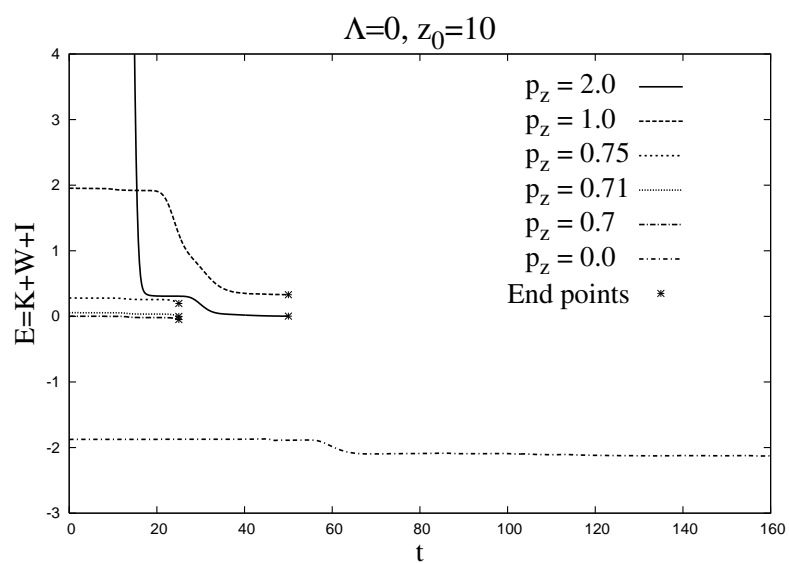

FIG. 4: The total energy $E=K+W+I$ for different initial parameters, all of them with $\Lambda=0$ and $z_{0}=10$. The configurations with $p_{z}=2.0,1.0$ start evolving with high values of the total energy, and show solitonic behavior; the total energy tends to zero once the density of probability (the partciles) gets out of the numerical domain, which happens by the time indicated with the star for such run. The configuration with $p_{z}=0$ remains with clearly negative total energy, so that the system is bound and the system collides, in the sense that there is no solitonic behavior and instead the two blobs get glued and remain like that. We are unable to conclude anything about the borderline cases. The stars at the end of borderline cases indicate the point at which we stoped the runs. The reason is that the lenghtscale of such cases is pretty much that of our numerical domain, and one expects the truning points of the blobs to be at a distance of the order of the domain size. By the time indicated with a star, there is burst of particles, related more to the fact that the blobs are returning bach to the domain than to a burst of particles due to the relaxiation of a single blob or to the fact that the blobs are leaving completely the domain.

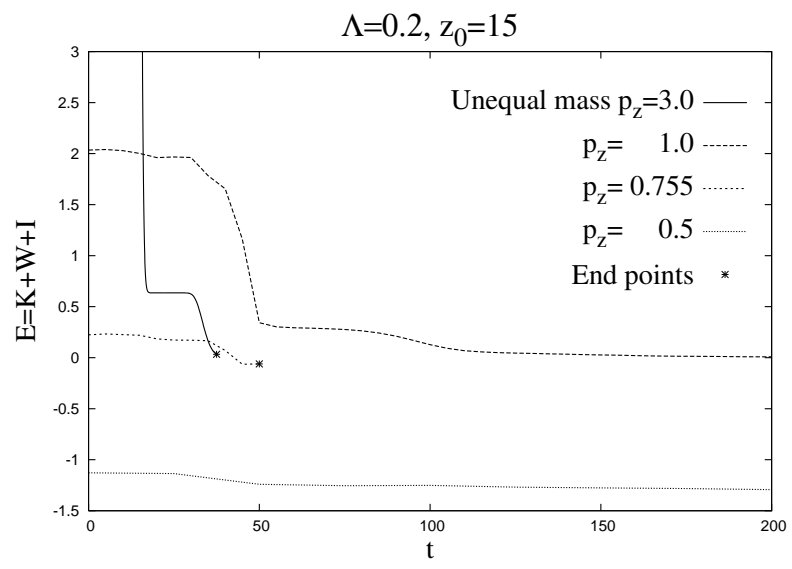

FIG. 5: The total energy $E=K+W+I$ for different initial parameters, all of them with $\Lambda=0.2$ and $z_{0}=15$. Once again, the configuration with a clearly positive total energy $\left(p_{z}=1.0\right.$ and the unequal mass case with $\left.p_{z}=3.0\right)$ shows solitonic behavior and those with clearly negative total energy $\left(p_{z} \leq 0.5\right)$ show a merger. Once againg, we stopped the run of the borderline case with $p_{z}=0.755$, which physical properties change at about $t \sim 50$, and the numerical domain used does not suffice to determine its fate. 


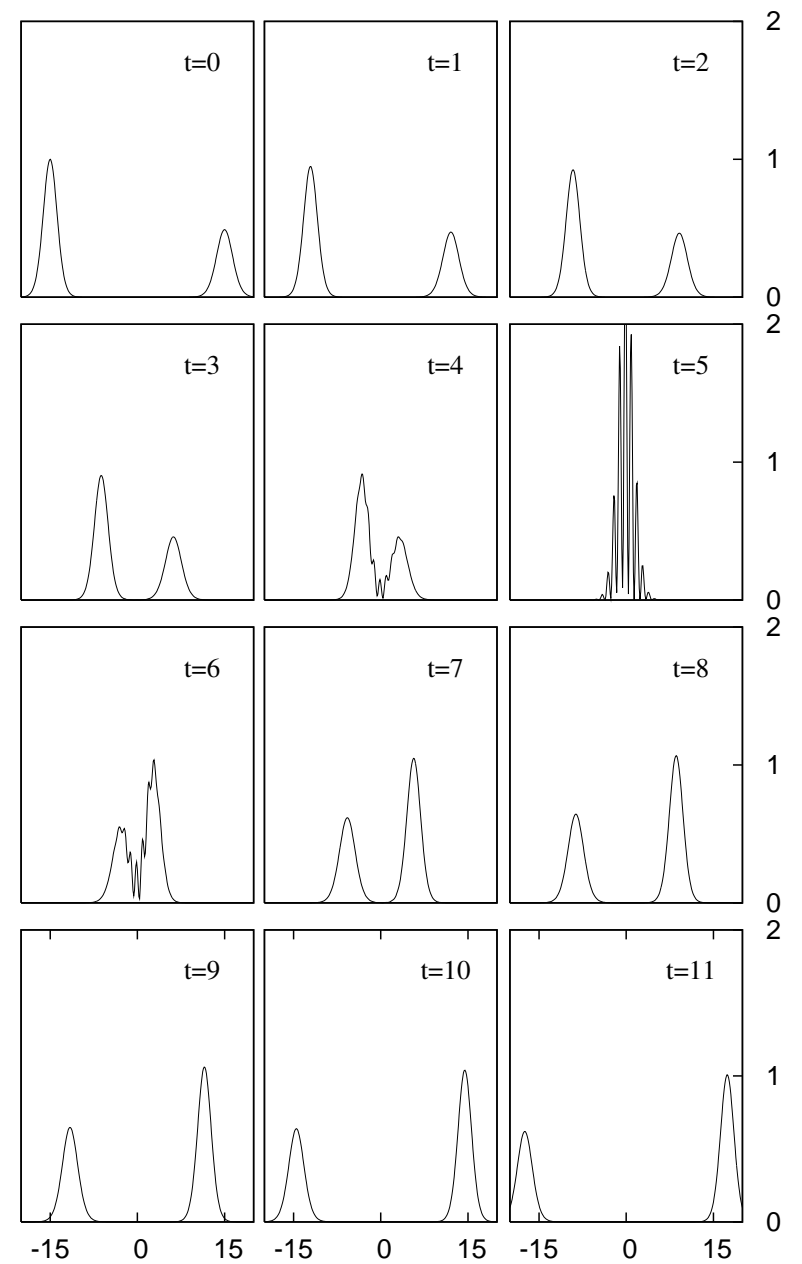

FIG. 6: Snapshots of the density of probability for the interaction between two unequal mass initial ground state configurations with $\Lambda=0.2, p_{z}=3.0$ and $z_{0}=15$ (the first case in the previous figure). The configuration shows solitonic behavior due to the fact that the total energy is always positive of the order of $E \sim 35$ until the blobs get absorbed by the sponge. The total energy remains positive all the time, until the blobs are absorbed by the sponge. The numerical domain used is $x \in[0,30]$ and $z \in[-30,30]$ with resolution $\Delta x=\Delta z=0.125$.

$8.3 \times 10^{6} \mathrm{yr}$; the maximum relative speed before the collision is $v \sim 830 \mathrm{~km} / \mathrm{s}$.

\section{CONCLUSIONS}

We have presented numerical solutions of the Schrödinger-Poisson system of equations which includes the non-linear term related to the self-interaction in the mean field Gross-Pitaevskii equation for Bose Condensates. In such case, the potential well is given by selfgravity of the density of probability of the system. The particular case we have studied corresponds to the inter-
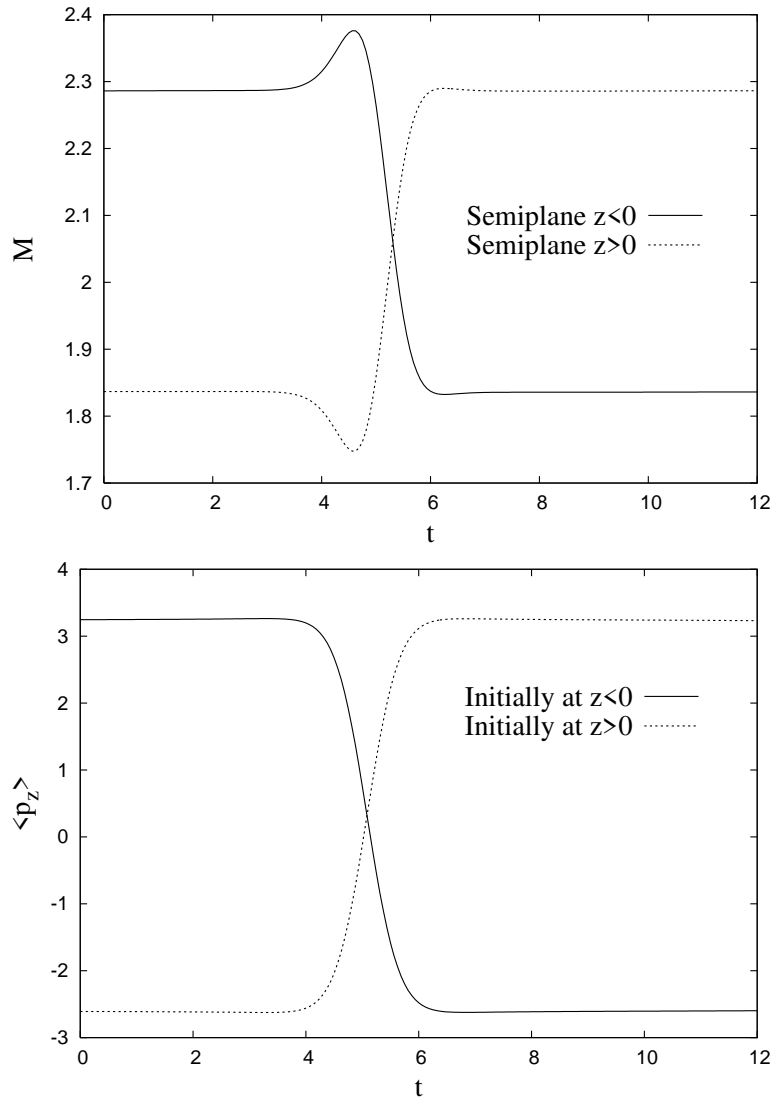

FIG. 7: Top: we show the transfer of particles from the $z<0$ half plane to the $z>0$ one and viceversa. The mass in each semiplane is shown. Bottom: we show the transfer of momentum between the two half planes, actually the expectation value calculated in each semiplane. The configuration evolved consists of two superposed ground state configurations with $\Lambda=0.2$, and respective central field values $\psi(0,0)=1,0.7$ and masses $M=2.437,1.9383$. The initial linear momentum is $p_{z}=3.0$. The density of probability reaches the edges of the numerical domain and vanishes. The total energy is always positive.

action between two ground state configurations (spherical both of them) [19].

We found that the initial blobs show solitonic behavior of the initial configuration, but that also the two configurations may collide. The system ends up colliding whenever the total energy of the system $E<0$ and the solitonic behavior appears when $E>0$. Unfortunately we can show this only for clearly non-zero values of the energy in each case and we cannot conclude anything about the borderline case, that is, when $E \sim 0$, because our simulations are unable to resolve the system for the quite long time needed and the spatial domain used.

Within the scalar field dark matter paradigm, the two initial blobs would represent two virialized structures made of dark matter. What we have shown is that not all couples of configurations are allowed to have a collision, and that the total energy would indicate whether or not 

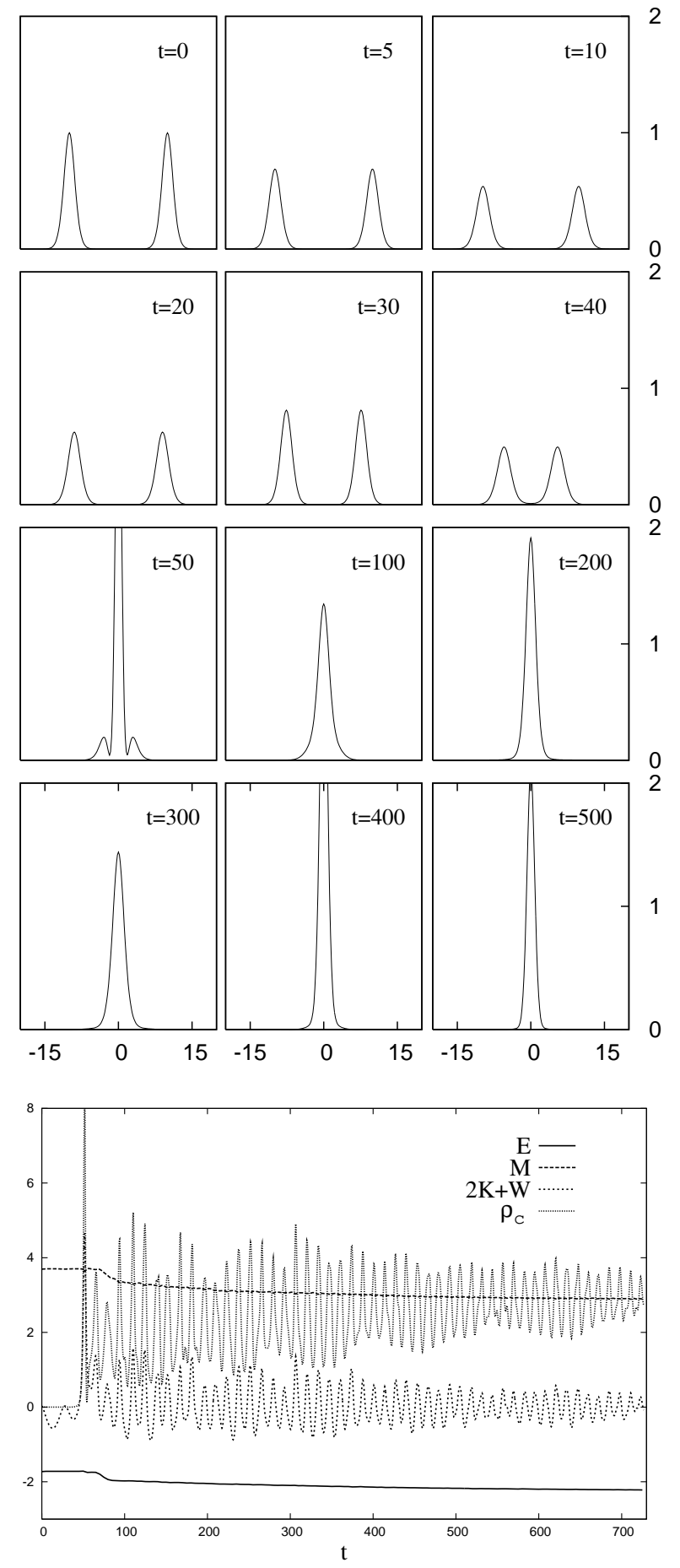

FIG. 8: Top: snapshots of the density of probability for an equal mass case with $\Lambda=0$ and initial parameters $p_{z}=0$, $z_{0}=10$. The system apparently is trapped within the gravitational potential well. Bottom: the central density, total energy, mass, and virial relation are shown. The run-time is not enough to decide whether the configuration will get virialized or not. However it behaves as a perturbed system: the total energy is approaching a constant value after a period of considerable activity, and the central density starts oscillating around a given value. a collision can occur. Our calculations also involve the presence of a self-interaction term in the scalar field, and therefore are within the Gross-Pitaevskii frame of Bose Condensates, which this time are gravitating.

\section{Acknowledgments}

This research is partly supported by projects CICUMSNH-4.9 and PROMEP-UMICH-PTC-121. The runs were carried out in the Ek-bek cluster of the "Laboratorio de Supercómputo Astrofísico (LASUMA)" at CINVESTAV-IPN. A. B. acknowledges support from CONACYT. 
[1] V. Sahni and L-M Wang, Phys. Rev. D 62, 103517 (2000).

[2] T. Matos and L. A. Ureña-López, Class. quantum Grav. 17, L75 (2000).

[3] T. Matos and L. A. Ureña-López, Phys. Rev. D 63, 063506 (2001).

[4] T. Matos and L. A. Ureña-López, Int. J. Mod. Phys. D13, 2287-2292 (2004).

[5] T. Matos, L. A. Ureña-López, M. Alcubierre, R. Becerril, F. S. Guzmán, D. Núñez, 2004, Lect. Notes. Phys. 646 , 401-420.

[6] F. S. Guzmán, T. Matos and H. Villegas-Brena, Astron. Nachr., 320, 97 (1999). F. S. Guzmán and T. Matos, Class. Quantum Grav. 17, L9 (2000). F. S. Guzmán and T. Matos, Rev. Mex. A. \& A. 37, 63-72 (2001). Matos T. Guzmán F. S., Nú nez D., 2000, Phys. Rev. D 62, 061301(R), ArXiv: astro-ph/0003398.

[7] J. P. Mbelek, Astron.Astrophys. 424, 761-764 (2004).

[8] E. W. Mielke and F. E. Schunck, Phys. Rev. D 66, 023503 (2002).

[9] F. E. Schunck, astro-ph/9802258.

[10] U. Nucamendi, M. Salgado and D. Sudarsky, Phys. Rev. D 63, 125016 (2001).

[11] F. S. Guzmán and T. Matos, Ann. Phys. (Leipzig), 9, SI-133 (2000). ArXiv: astro-ph/0002126.

[12] A. Arbey, J. Lesgourgues and P. Salati, Phys. Rev. D 64, 123528 (2001).

[13] A. Arbey, J. Lesgourgues and P. Salati, Phys. Rev. D 65, 083514 (2002).

[14] A. Arbey, J. Lesgourgues, and P. Salati, Phys. Rev. D 68, 023511 (2003).
[15] W. Hu, R. Barkana, and A. Gruzinov, Phys. Rev. Lett. 85, 1158-1161 (2000).

[16] M. Alcubierre, F. S. Guzmán, T. Matos, D. Núñez, L. A. Ureña-López and P. Wiederhold, Class. Quantum Grav. 19, 5017 (2002). ArXiv: gr-qc/0110102.

[17] M. Alcubierre, R. Becerril, F. S. Guzmán, T. Matos, D. Núñez and L. A. Ureña-López, Class. Quantum Grav. 20, 2883 (2003). ArXiv: gr-qc/0301105.

[18] F. S. Guzmán and L. A. Ureña-López, Phys. Rev. D 68, 024023 (2003). ArXiv: astro-ph/0303440.

[19] F. S. Guzmán and L .A. Ureña-López, ApJ 645, 814 (2006). ArXiV: astro-ph/0603613.

[20] A. Bernal and F. S Guzmán, Phys. Rev. D 74, 063504 (2006). ArXiv: astro-ph/0608523.

[21] L. P. Pitaevskii, Zh. Eksp. Teor. Fyz. 40 (1961) 646. Sov. Phys, JETP, 13 (1961) 451. E. P. Gross, Nouvo Cimento 20 (1961) 454.

[22] F. S. Guzmán, Phys. Rev. D 73021501 (2006). ArXiV: gr-qc/0512081.

[23] F. S. Guzmán and L. A. Ureña-López, Phys. Rev. D 69, 124033 (2004). ArXiv: gr-qc/0404014.

[24] D. I. Choi, Phys. Rev A 66, 063609 (2002).

[25] Harrison R., Moroz I., Tod K. P., 2002, mathphys/0208046.

[26] Smith G. D., 1965. Numerical Solution of Partial Differential Equations, Oxford University Press.

[27] M. Israeli and S. A. Orszag, J. Comp. Phys. 41, 115 (1981)

[28] X. Z. Wang, Phys. Rev. D 64, 124009 (2001). 